\title{
Computational modeling of motile cilia generated cerebral flow dynamics in zebrafish embryo
}

\author{
Huseyin Enes Salman ${ }^{1}$, Natalie Jurisch-Yaksi ${ }^{2}$, Huseyin Cagatay Yalcin ${ }^{1}$

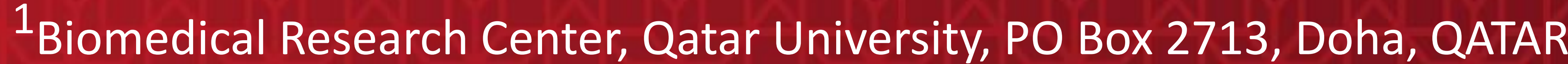 \\ ${ }^{2}$ Faculty of Medicine and Health Sciences, Norwegian University of Science and Technology, Trondheim, NORWAY
}

\begin{tabular}{|c|}
\hline BACKGROUND \\
\hline $\begin{array}{l}\text { - Motile cilia are hair-like microscopic structures which move the fluids } \\
\text { along the epithelial surfaces. } \\
\text { - Cilia cover a wide range of regions in the nervous system, such as the } \\
\text { nasal cavity, central canal of spinal cord, and brain ventricles. } \\
\text { - Motile cilia-driven cerebrospinal fluid (CSF) flow in the brain ventricles } \\
\text { has an important role in brain development. } \\
\text { - Embryos lacking motile cilia develop neurological defects due to } \\
\text { altered CSF flow. }\end{array}$ \\
\hline
\end{tabular}

\section{OBJECTIVE}

To investigate the effect of motile-cilia motion on the altered CSF flow, and to understand the role of CSF flow in the brain development and physiology.

\section{METHODOLOGY}

\section{Computational fluid dynamics simulations}

- Mathematical solution is approximated with numerical techniques by following the steps:

- Generation of 2-dimensional simplified brain ventricle flow domain

- Discretization of the problem domain into finite elements (Meshing)

- Defining a full cycle of motile-cilia motion using the time-lapse microscopic movies showing movements of a fluorescently labeled motile-cilia in a zebrafish embryo (48-hour post-fertilization)

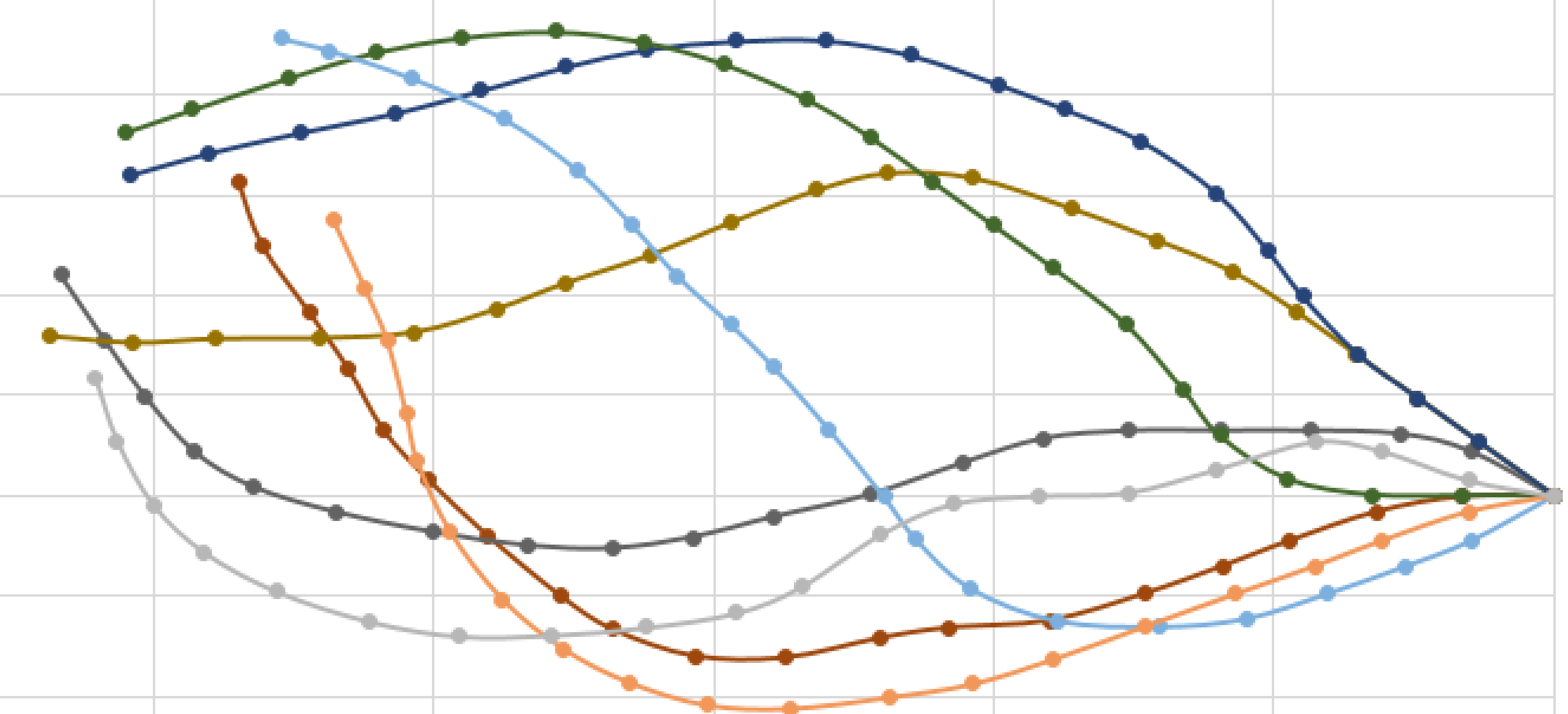

Motile-cilia motion steps during one full cycle

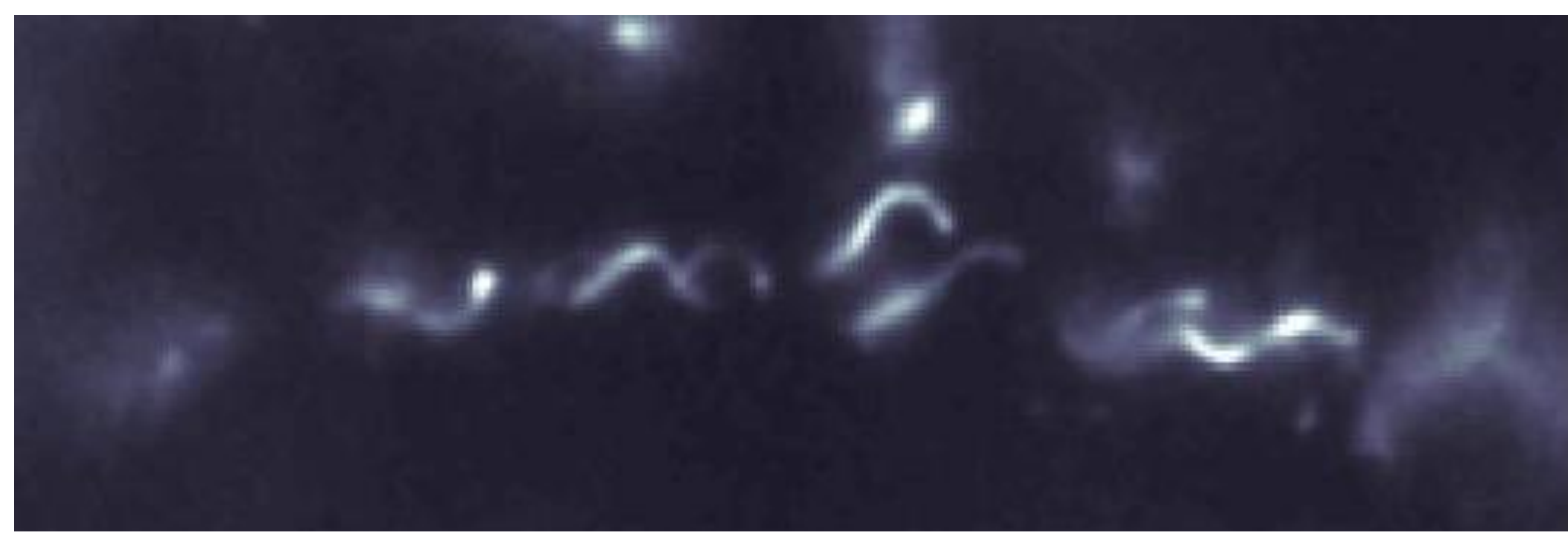

Motile-cilia in the brain ventricle of 48 hpf zebrafish embryo

- Post-processing the results after 30 cycles of motile-cilia motion

- The governing equations in fluid domain:

$$
\begin{gathered}
\rho_{f} \frac{\partial \mathbf{v}}{\partial t}+\rho_{f}(\mathbf{v}-\mathbf{w}) \cdot \nabla \mathbf{v}-\nabla \cdot \boldsymbol{\tau}_{f}=\mathbf{f}_{f}^{\mathrm{B}} \\
\nabla \cdot \mathbf{v}=0
\end{gathered}
$$

$\rho_{f}$ : Mass density of fluid, $\mathbf{v}$ : Fluid velocity vector, $t$ : Time $\mathbf{w}$ : Velocity of the fluid domain (i.e. moving coordinate velocity)

$\boldsymbol{\tau}_{f}$ : Fluid stress tensor, $\quad \mathbf{f}_{f}^{\mathrm{B}}$ : Body forces

- CSF flow velocities are determined in the entire flow domain.

- The effects on the generated flow are elucidated by investigating the cilia beating angle, multiple cilia formations, and phase difference between different ciliary beats.

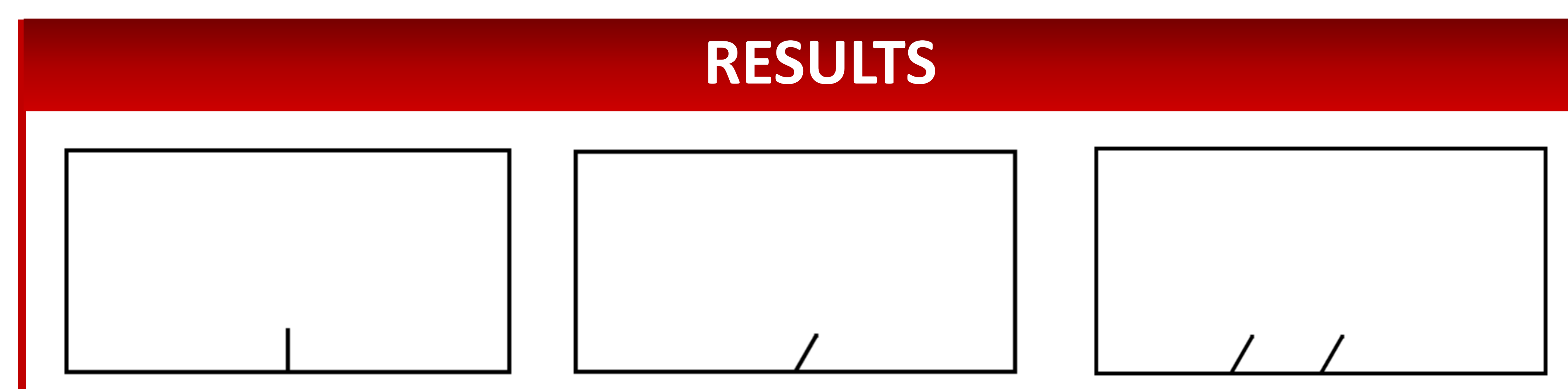

Cilia with $90^{\circ}$ angle

Cilia with $60^{\circ}$ angle

Multiple cilia with $60^{\circ}$ angle

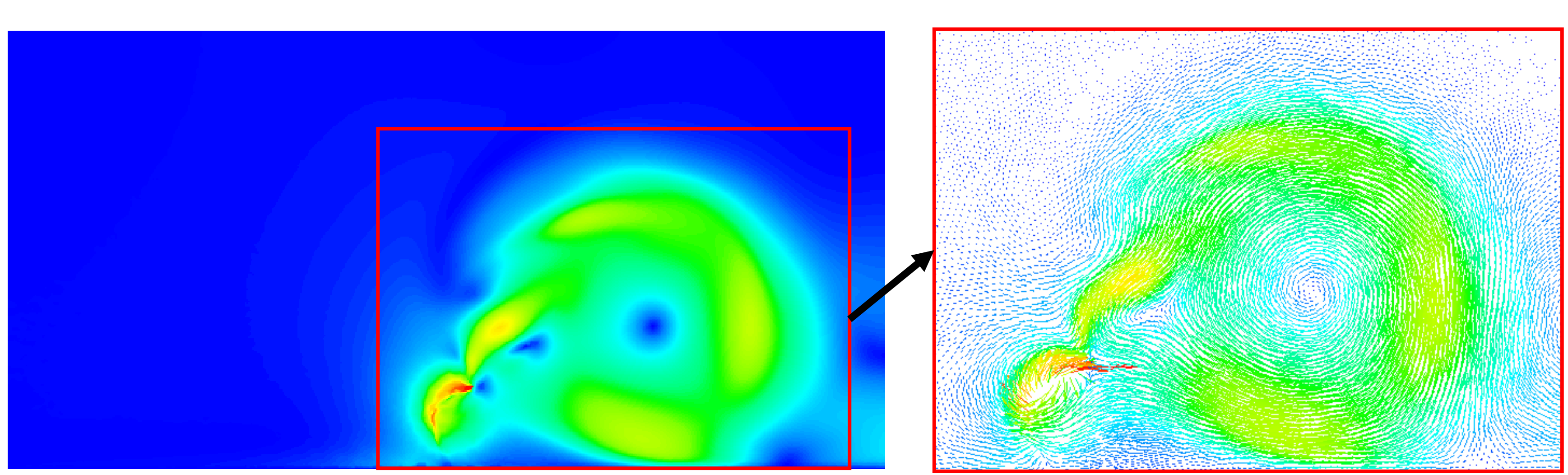

$90^{\circ}$ angle between the cilia and brain ventricle wall
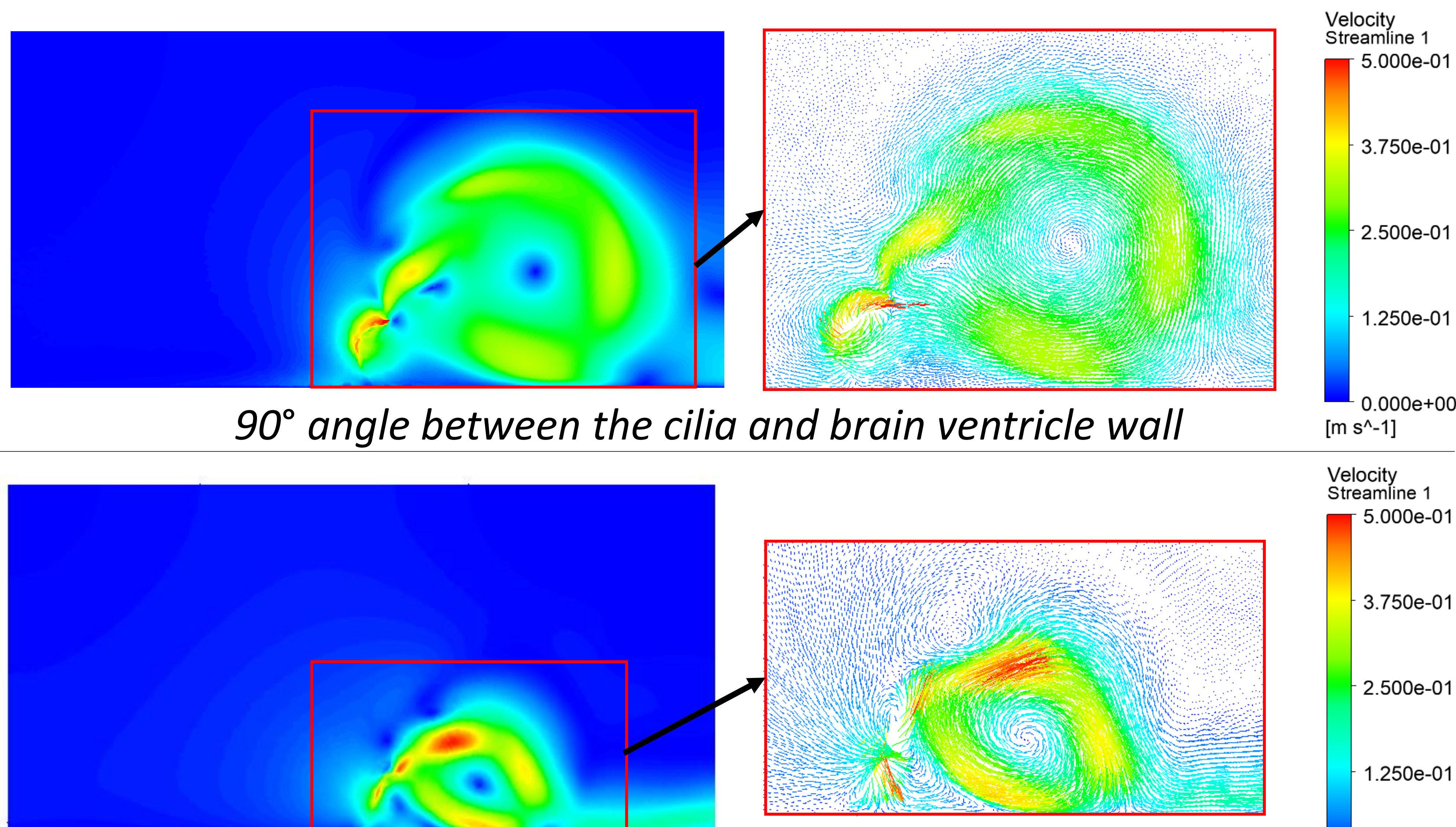

$70^{\circ}$ angle between the cilia and brain ventricle wall
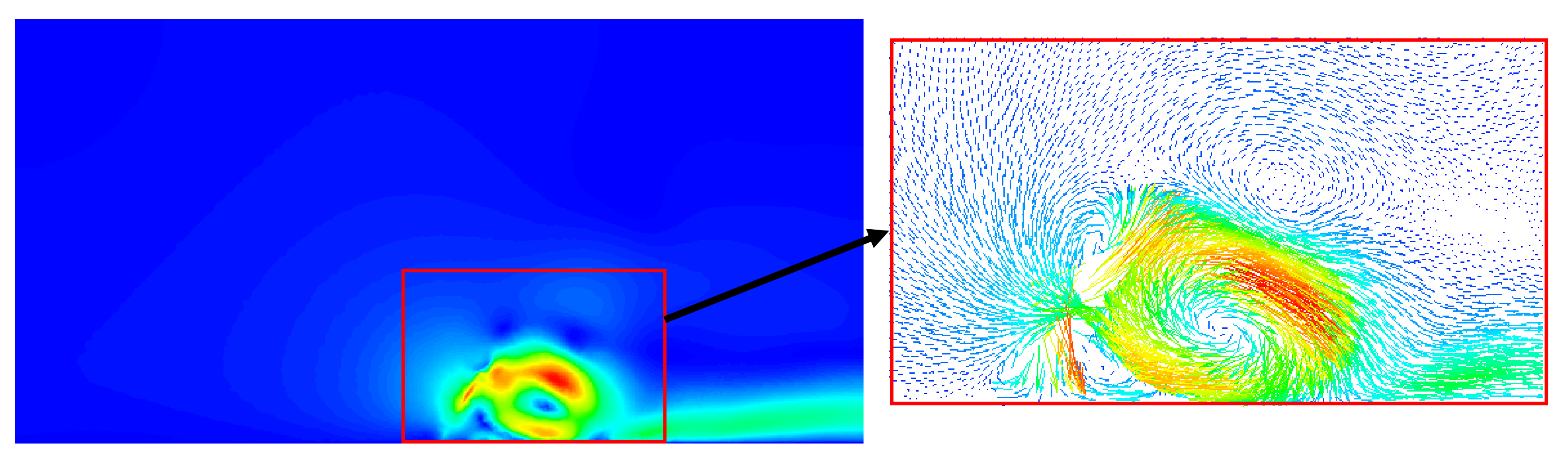

$60^{\circ}$ angle between the cilia and brain ventricle wall
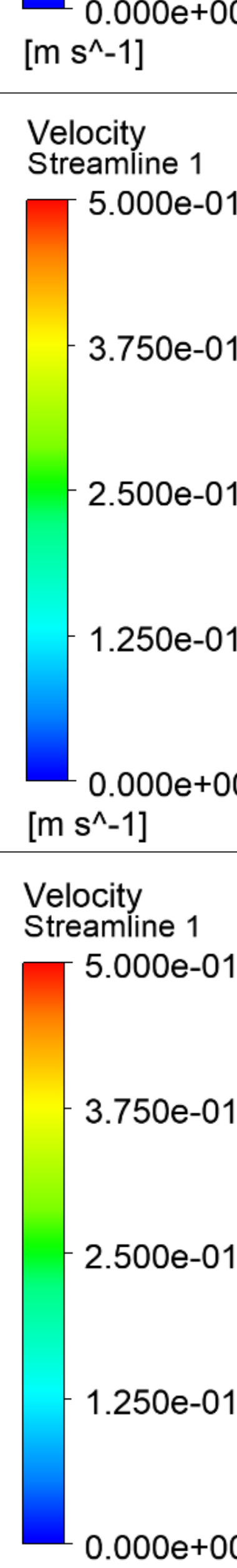

$\frac{0.0000+00}{\left[m s^{-11]}\right.}$

- Ciliary beating generated a directional flow in the form of a circulating vortex.

- The angle of ciliary beating significantly affected the flow velocity.

- As the angle between the wall and cilia decreases, CSF flow achieves higher velocities ( $8.4 \%$ difference between $90^{\circ}$ and $60^{\circ}$ cilia angles).

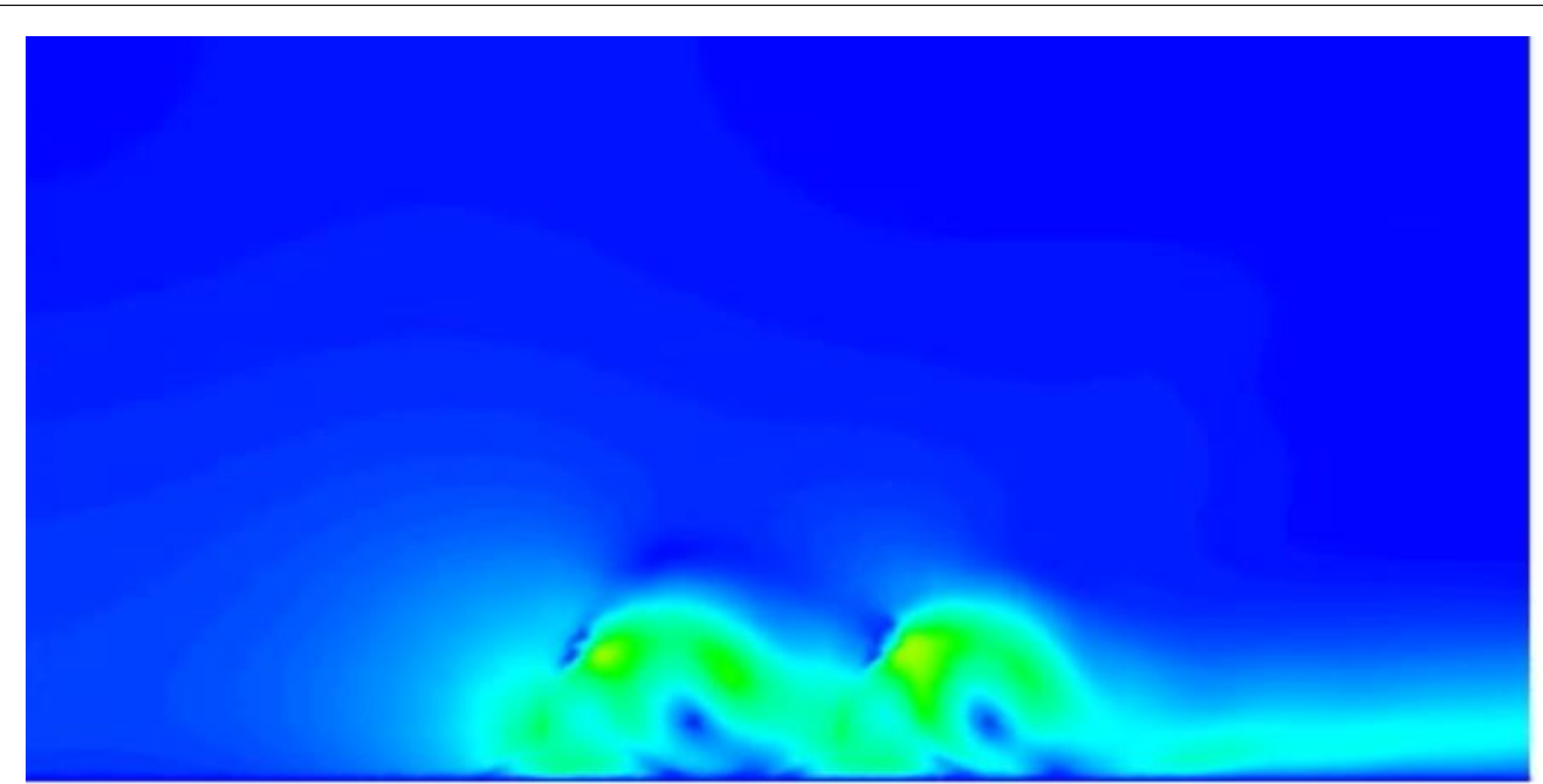

$60^{\circ}$ angle between in-phase multiple cilia and brain ventricle wall

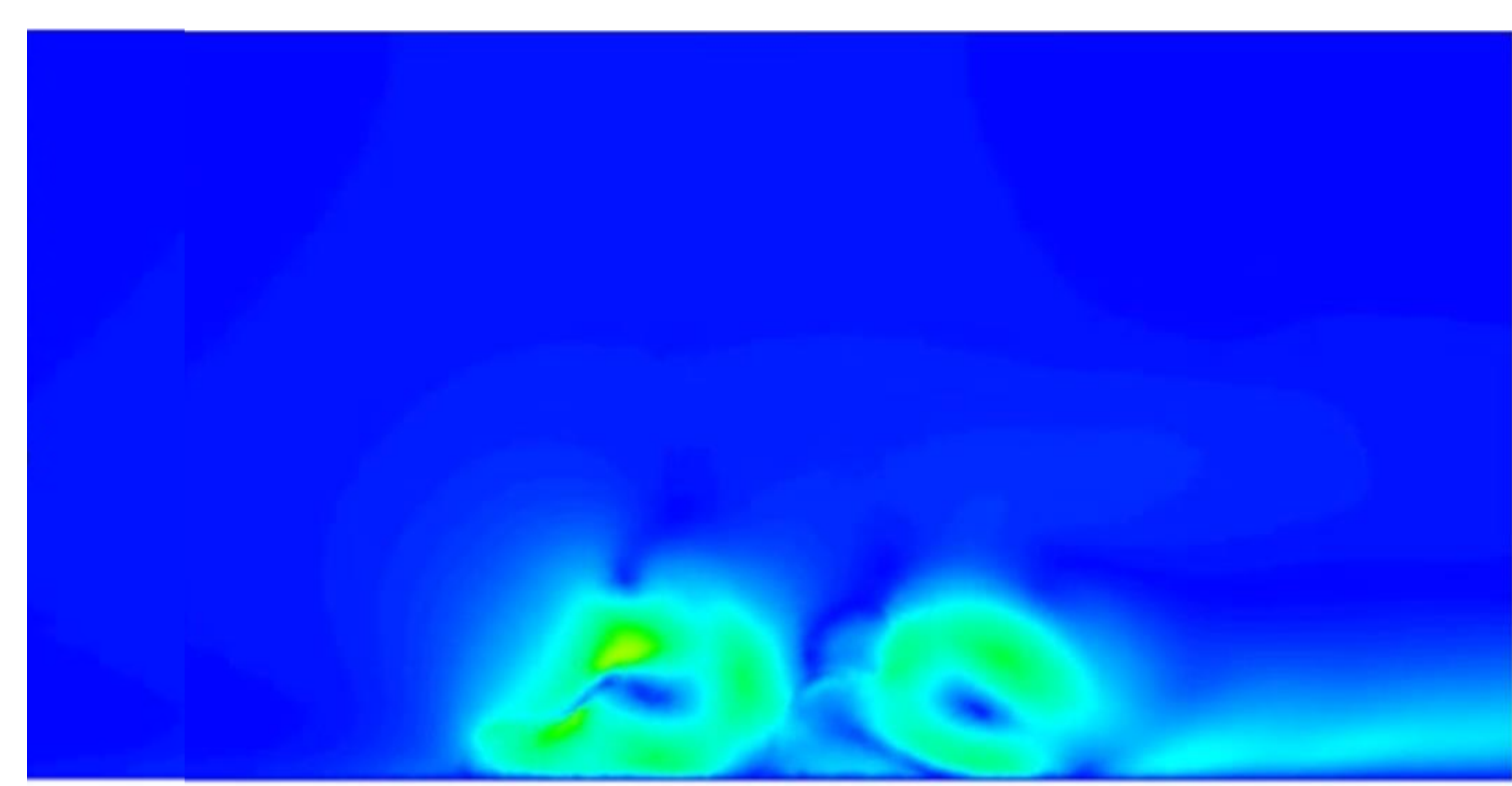

$60^{\circ}$ angle between out-of-phase multiple cilia and brain ventricle wall
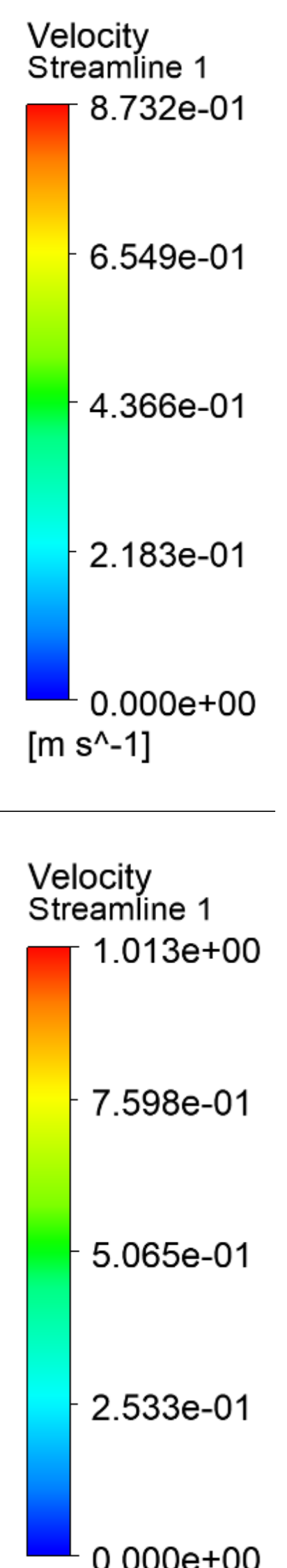

Multiple cilia formations increased the flow velocity but the significance of multiple cilia is not as critical as the beating angle.

- Interestingly, phase difference between the multiple cilia beats increased the directional flow velocity (16.0\% difference with in-phase cilia beating).

\section{CONCLUSION}

Motile-cilia generated flow dynamics are investigated, and it is concluded that out-of-phase multiple ciliary beating is the optimum form of beating in order to generate a directional flow. 